Published in final edited form as:

Acta Neuropathol. 2010 June ; 119(6): 669-678. doi:10.1007/s00401-010-0667-0.

\title{
Novel CSF biomarkers for Alzheimer's disease and mild cognitive impairment
}

\author{
William T. Hu, \\ Department of Neurology, University of Pennsylvania School of Medicine, Philadelphia, PA, USA \\ Center for Neurodegenerative Disease Research, University of Pennsylvania School of Medicine, \\ HUP, Maloney 3rd Floor, 36th and Spruce Streets, Philadelphia, PA 19104-4283, USA, \\ trojanow@mail.med.upenn.edu
}

\section{Alice Chen-Plotkin,}

Department of Neurology, University of Pennsylvania School of Medicine, Philadelphia, PA, USA

Center for Neurodegenerative Disease Research, University of Pennsylvania School of Medicine, HUP, Maloney 3rd Floor, 36th and Spruce Streets, Philadelphia, PA 19104-4283, USA

\section{Steven E. Arnold,}

Department of Psychiatry, University of Pennsylvania School of Medicine, Philadelphia, PA, USA

Murray Grossman,

Department of Neurology, University of Pennsylvania School of Medicine, Philadelphia, PA, USA

Christopher M. Clark,

Department of Neurology, University of Pennsylvania School of Medicine, Philadelphia, PA, USA

Leslie M. Shaw,

Department of Pathology and Laboratory Medicine, University of Pennsylvania School of Medicine, HUP, Maloney 3rd Floor, 36th and Spruce Streets, Philadelphia, PA 19104-4283, USA

Eve Pickering,

Pfizer Global Research and Development, Groton, CT, USA

Max Kuhn,

Pfizer Global Research and Development, Groton, CT, USA

Yu Chen,

Pfizer Global Research and Development, Groton, CT, USA

Leo McCluskey,

Department of Neurology, University of Pennsylvania School of Medicine, Philadelphia, PA, USA

Lauren Elman,

Department of Neurology, University of Pennsylvania School of Medicine, Philadelphia, PA, USA

Jason Karlawish,

Department of Medicine, University of Pennsylvania School of Medicine, Philadelphia, PA, USA

Howard I. Hurtig,

\footnotetext{
(C) Springer-Verlag 2010

Correspondence to: William T. Hu; John Q. Trojanowski.

Present Address: W. T. Hu, Department of Neurology, Emory University School of Medicine, Atlanta, GA, USA

Electronic supplementary material The online version of this article (doi:10.1007/s00401-010-0667-0) contains supplementary material, which is available to authorized users.
} 
Department of Neurology, University of Pennsylvania School of Medicine, Philadelphia, PA, USA

Andrew Siderowf,

Department of Neurology, University of Pennsylvania School of Medicine, Philadelphia, PA, USA

Virginia M.-Y. Lee,

Center for Neurodegenerative Disease Research, University of Pennsylvania School of Medicine, HUP, Maloney 3rd Floor, 36th and Spruce Streets, Philadelphia, PA 19104-4283, USA

Department of Pathology and Laboratory Medicine, University of Pennsylvania School of Medicine, HUP, Maloney 3rd Floor, 36th and Spruce Streets, Philadelphia, PA 19104-4283, USA

Institute on Aging, University of Pennsylvania School of Medicine, Philadelphia, PA, USA

Holly Soares, and

Pfizer Global Research and Development, Groton, CT, USA

John Q. Trojanowski

Center for Neurodegenerative Disease Research, University of Pennsylvania School of Medicine, HUP, Maloney 3rd Floor, 36th and Spruce Streets, Philadelphia, PA 19104-4283, USA

Department of Pathology and Laboratory Medicine, University of Pennsylvania School of Medicine, HUP, Maloney 3rd Floor, 36th and Spruce Streets, Philadelphia, PA 19104-4283, USA

Institute on Aging, University of Pennsylvania School of Medicine, Philadelphia, PA, USA

\section{Abstract}

Altered levels of cerebrospinal fluid (CSF) peptides related to Alzheimer's disease (AD) are associated with pathologic AD diagnosis, although cognitively normal subjects can also have abnormal levels of these AD biomarkers. To identify novel CSF biomarkers that distinguish pathologically confirmed $\mathrm{AD}$ from cognitively normal subjects and patients with other neurodegenerative disorders, we collected antemortem CSF samples from $66 \mathrm{AD}$ patients and 25 patients with other neurodegenerative dementias followed longitudinally to neuropathologic confirmation, plus CSF from 33 cognitively normal subjects. We measured levels of 151 novel analytes via a targeted multiplex panel enriched in cytokines, chemokines and growth factors, as well as established AD CSF biomarkers (levels of A $\beta 42$, tau and p-tau 181 ). Two categories of biomarkers were identified: (1) analytes that specifically distinguished AD (especially CSF A $\beta 42$ levels) from cognitively normal subjects and other disorders; and (2) analytes altered in multiple diseases (NrCAM, PDGF, C3, IL-1 $\alpha$ ), but not in cognitively normal subjects. A multiprong analytical approach showed AD patients were best distinguished from non-AD cases (including cognitively normal subjects and patients with other neurodegenerative disorders) by a combination of traditional AD biomarkers and novel multiplex biomarkers. Six novel biomarkers (C3, CgA, IL-1 $\alpha$, I-309, NrCAM and VEGF) were correlated with the severity of cognitive impairment at CSF collection, and altered levels of IL- $1 \alpha$ and TECK associated with subsequent cognitive decline in 38 longitudinally followed subjects with mild cognitive impairment. In summary, our targeted proteomic screen revealed novel CSF biomarkers that can improve the distinction between AD and non-AD cases by established biomarkers alone.

\section{Keywords}

Amyloid beta; Abeta42; Diagnosis; IL-1 $\alpha$; MCI; NrCAM; PDGF; Resistin; TECK; TDP-43; Tau 


\section{Introduction}

Alzheimer's disease (AD), frontotemporal lobar degenerations (FTLD) and dementia with Lewy bodies (DLB) are major neurodegenerative disorders pathologically characterized by lesions composed of disease-specific misfolded proteins. Their clinical syndromes often have overlapping features, making antemortem prediction of pathology challenging. Yet, as specific disease-modifying therapies become available, it is increasingly important that such diagnoses be made. Analytes in cerebrospinal fluid (CSF) associated with AD pathology, such as total tau, tau phosphorylated at threonine 181 (p-tau 181 ) and A $\beta 1-42$ (or A $\beta 42$ ), offer the potential for more accurate diagnosis, although cognitively normal elderly subjects could have altered levels of these traditional AD biomarkers $[20,24,26]$. Peptides in common inflammatory and apoptotic pathways, growth factors and other analytes have also been proposed as novel CSF biomarkers for $\mathrm{AD}$ [23] and the use of novel biomarkers on their own or in conjunction with traditional AD biomarkers may improve the specificity of CSF-based AD diagnosis. Recently, a proteomic approach targeting specific inflammatory and growth factors in the plasma identified novel biomarkers for the clinical diagnosis of $\mathrm{AD}$ [21], but the absence of pathological confirmation makes these results difficult to interpret since 10-20\% of patients clinically diagnosed with $\mathrm{AD}$ are found on autopsy to have a cause for dementia other than $\mathrm{AD}$. The determination of diagnostic accuracy for novel AD biomarkers thus requires studies of biofluids obtained during life from well-characterized $\mathrm{AD}$ patients longitudinally followed to autopsy confirmation [1,3,7]. Also, novel CSF biomarkers for AD can potentially facilitate disease staging and predict rates of clinical decline, as these biomarkers could represent factors that modulate AD pathogenesis associated with various stages of the disease. Characterization of a select panel of CSF biomarkers can, therefore, be critical in diagnosis and prognosis, and alterations in their levels can be further considered as secondary endpoints in future therapeutic trials.

Here, we tested the hypothesis that distinct sets of CSF peptides and proteins are associated with $\mathrm{AD}$ in contrast to cognitively normal subjects and other common neurodegenerative disorders, including FTLD with TDP-43-immunoreactive lesions (FTLD-TDP), FTLD with tau-immunoreactive inclusions (FTLD-Tau), and DLB. We collected and analyzed CSF samples antemortem from a total of 162 subjects, and the concentrations of 151 analytes in the Rules Based Medicine Human DiscoveryMAP ${ }^{\mathrm{TM}}$ panel (referred to below here as MAP) were measured by a Luminex-based multiplex platform. As the choice of analytical strategy in this type of high-dimensional data may significantly alter the composition of the identified biomarker panel, we analyzed the same body of data through three independent algorithms. Alterations in the levels of these candidate biomarkers were first analyzed according to the traditional statistical modeling, including Mann-Whitney $U$ test and logistic regression. To avoid bias associated with feature pre-selection by univariate analysis and instability of linear models associated with high-dimensional data, we searched for novel AD biomarkers by two additional methods: a tree-based classification algorithm (random forest) and a nearest shrunken centroid algorithm (predictive analysis of microarrays, or PAM) [21,29]. The diagnostic accuracy of novel analyte combinations predicted by each algorithm was then assessed. Lastly, novel AD biomarkers were then evaluated for their relationship to the severity of cognitive impairment in $\mathrm{AD}$, and their potential role in predicting rates of cognitive decline in patients with mild cognitive impairment (MCI).

\section{Materials and methods}

\section{Participants}

Patients and control subjects were recruited and longitudinally followed at Penn in specialty services dedicated to the evaluation and management of neurodegenerative diseases (Supplementary Table 1). All protocols were approved by the Penn Institutional Review Board. 
Each patient in the autopsy cohort had undergone detailed cognitive, neurological, neuroimaging and laboratory examinations to ensure the accuracy of clinical diagnosis according to established criteria for AD [6], frontotemporal dementia (FTD) [16], amyotrophilc lateral sclerosis (ALS) [22] and DLB [13]. Autopsy-confirmed cases of AD $(n=66)$, FTLD $(n=16)$ and DLB $(n=2)$ were characterized neuropathologically with detailed immunohistochemical analysis for pathology associated with each major neurodegenerative disorder, including A 342 , hyperphosphorylated tau, hyperphosphorylated TDP-43 and alphasynuclein as described by Neumann et al. [18]. Seven patients with clinical FTD-ALS, but no autopsy was added to the FTLD-TDP group, as these cases nearly always have TDP-43 pathology. Thirty-eight patients with MCI were also recruited to assess predictors of cognitive decline. Each MCI patient was diagnosed by modified Petersen criteria [30], and followed longitudinally with serial cognitive and neurological examination. Cognitively normal subjects were evaluated at the time of CSF collection, and continued to undergo annual testing to confirm their cognitive status. ApoE genotyping was performed for all subjects (Supplementary Material).

\section{Procedures}

Baseline CSF samples were obtained during routine diagnostic lumbar puncture as previously described [3,24]. Briefly, lumbar puncture was performed with a 20- or 24- gauge spinal needle, and CSF was transferred into polypropylene tubes. At the time of CSF collection, aliquots ( 0.5 $\mathrm{mL}$ ) were prepared, bar-coded and then stored in polypropylene vials at $-80^{\circ} \mathrm{C}$ until analysis (mean 8.7 years, $\mathrm{SD}=3.6$ years). Samples were then grouped altogether and simultaneously interrogated by Rules-Based Medicine, Inc. (Austin, TX) for levels of 151 analytes using the Human DiscoveryMAPTM panel and a Luminex 100 platform (Supplementary Material). The 151 MAP analytes were assembled by RBM into pre-formatted assays that RBM designed for studies of a number of different diseases including cancer, autoimmune disorders and AD based on the previous associations with $\mathrm{AD}$ of many, but by no means all of these analytes in peerreviewed literature. Measures of CSF A 442 , total tau and $p$-tau ${ }_{181}$, were performed using the multiplex xMAP Luminex platform (Luminex Corp, Austin, TX) with Innogenetics (INNOBIA AlzBio3, Ghent, Belgium) immunoassay kit-based reagents as described [24].

\section{Statistical analysis}

Statistical analysis was performed in SPSS 12.0, Random Forests (http://www.stat.berkeley.edu/ breiman/RandomForests/) and SAM/PAM. For testing of stability associated with each analyte, Pearson's correlation analysis was performed between analyte levels and time in $-80^{\circ} \mathrm{C}$ storage. For each analytical strategy, diagnostic performance (sensitivity, specificity, accuracy) was determined using traditional AD biomarkers alone (tau, $\mathrm{p}$-tau $\left.{ }_{181}, \mathrm{~A} \beta 42\right)$, MAP biomarkers alone or both traditional and MAP biomarkers. For each model, performance characteristics reported were based on the cross-validation. In the first model (Model 1), analytes that differed significantly between cognitively normal and AD by Mann-Whitney $U$ test (nominal $P<0.01$ ) were entered into logistic regression models for AD identification, adjusting for age and gender. Sensitivity and specificity of Model 1 were obtained by leave-one-out approach in discriminant analysis. In random forest analysis, analytes were entered into the analysis with nodes optimized for best classification of AD versus cognitively normal (Model 2). Out-of-box error rate was used to derive diagnostic accuracy, with sensitivity and specificity derived from the confusion matrix. In PAM, analytes that significantly differentiated AD from cognitively normal were identified, and diagnostic accuracy was derived through internal cross-validation (Model 3). Given the number of analytes relative to the number of subjects, interaction terms were not entered in the logistic regression model (Model 1). Random forest analysis (Model 2) and PAM (Model 3) each relies less on the assumption of normal distribution and takes into account possible correlations between analytes, although each algorithm can derive different analytes to account for 
variations in the respective classification model. Thus, to expand our analysis beyond the strengths and constraints of any one algorithm, we sought to identify biomarkers determined by at least two of these three well-established analytical strategies as key novel biomarkers. A similar three-approach strategy was employed to determine biomarkers that distinguished between $\mathrm{AD}$ and non- $\mathrm{AD}$ neurodegenerative disorders.

For cross-sectional association between novel AD biomarker levels and severity of cognitive impairment at the time of CSF collection, Pearson's correlation coefficient was used to relate levels of newly identified CSF AD biomarkers with cognitive performance characterized by Mini-Mental Status Examination (MMSE) in autopsyconfirmed AD cases. For correlation of CSF biomarker levels and rates of cognitive decline following CSF collection in MCI, rates of cognitive decline were first estimated by the slope of MMSE score linear regression over time. Pearson's correlation coefficient was then determined for CSF biomarker levels and rates of cognitive decline. Effects from age and gender were adjusted for all diagnostic and progression models.

\section{Results}

All CSF was obtained from patients with informed consent as described [2,12,24]. Levels of 151 analytes in the MAP were measured in the CSF, with 106 analytes having measurable levels for analysis (Supplementary Table 2). Four analytes (angiotensinogen, BMP-6, endothelin-1, SGOT) demonstrated level changes that corresponded to time stored in $-80^{\circ} \mathrm{C}$ freezer and were excluded from the analysis because of their apparent instability with increasing length of storage. To determine the best biomarkers of $\mathrm{AD}$, we used three independent analytical strategies to identify MAP analytes associated with $\mathrm{AD}$, and combined traditional AD biomarkers and MAP analytes to identify complementary AD biomarkers.

\section{$A D$ versus cognitively normal}

In Model 1, 21 MAP analytes were found to differ between cognitively normal subjects and AD (Fig. 1) by Mann-Whitney $U$ test at $P<0.01$, and only a minority of these were specifically changed in $\mathrm{AD}$, including resistin and thrombospondin-1. MAP analytes alone, but not traditional AD biomarkers, were entered into a forward stepwise logistic regression model (Table 1). Leave-one-out discriminant analysis using the five resultant MAP analytes achieved $84.8 \%$ sensitivity and $87.9 \%$ specificity, with overall $85.9 \%$ accuracy. By comparison, traditional AD biomarkers A 342 and total tau yielded greater sensitivity (92.4\%), but less specificity $(81.8 \%)$ for overall accuracy of $88.9 \%$. Combining MAP analytes and traditional $\mathrm{AD}$ biomarkers resulted in a model differentiating $\mathrm{AD}$ from cognitively normal subjects by the following biomarkers: levels of tau, A $\beta 42$, complement 3 (C3), neuron-glia-CAM-related cell adhesion molecule (NrCAM) and platelet-derived growth factor (PDGF, Table 1). This combined model has high sensitivity (97.0\%) and specificity (93.9\%) with $96.0 \%$ accuracy, and improved upon the traditional $\mathrm{AD}$ model by correctly reclassifying up to four cognitively normal subjects with pathologic CSF levels of tau and A $\beta 42$, and three AD subjects with nonpathologic levels of CSF tau and A $\beta 42$.

Feature pre-selection and the lack of an independent validation set may bias our classification results. Hence, we performed a similar analysis of $\mathrm{AD}$ versus cognitively normal through random forest (Model 2) and PAM (Model 3) using age, gender and levels of 3 traditional biomarkers and $106 \mathrm{MAP}$ analytes, as each analysis incorporates internal cross-validation that is more objective than leave-one-out analysis. Model 2 using MAP analytes alone identified some analytes from Model 1, including C3, fatty acid-binding protein (Fabp), IL-23, NrCAM and PDGF, among others (Table 2; Fig. 2a). The out-of-box error rate of traditional AD biomarkers was $12.1 \%$, which reduced to $6.1 \%$ when MAP analytes were introduced with 93.9\% accuracy. Model 3 also identified NrCAM and PDGF as important biomarkers useful 
in distinguishing between $\mathrm{AD}$ and cognitively normal subjects (Tables 3, 4; Fig. 2a). Diagnostic accuracy obtained through cross-validation was $93.9 \%$ in Model 3. A summary of analytes important in distinguishing between $\mathrm{AD}$ and cognitive normal subjects is shown in Fig. 2a, including $\mathrm{A} \beta 42$, tau, $\mathrm{NrCAM}$ and PDGF identified by all three algorithms.

\section{AD versus other neurodegenerative disorders}

With the emergence of substrate-specific therapeutic interventions, it is critically important to identify biomarkers that reliably differentiate the major neurodegenerative disorders from one another. To this end, we assessed which CSF biomarkers best differentiated AD from other neurodegenerative disorders using a similar series of analytical strategies.

Among traditional AD biomarkers, $\mathrm{A} \beta 42$ and $\mathrm{p}$-tau ${ }_{181}$ levels discriminated between $\mathrm{AD}$ and non-AD neurodegenerative disorders in all models. Among novel MAP analytes, agoutirelated peptide (AgRP) was identified by all algorithms to distinguish between $\mathrm{AD}$ and nonAD disorders (Fig. 2b). Post hoc analysis showed AgRP as most altered in FTLD-TDP (Fig. 3 ) and its classification power may rest in identifying FTLD-TDP cases. Tau, eotaxin-3 and hepatocyte growth factor (HGF) were additionally identified by both RF and PAM to be important in distinguishing between $\mathrm{AD}$ and non-AD disorders (Fig. 2b). Similar to the classification role of AgRP, eotaxin-3 was most different between AD and FTLD-TDP $(P=$ $0.001)$, and HGF was most different between AD and FTLD-Tau $(P=0.002$, both comparisons by Mann-Whitney $U$ test; Fig. 3). Thus, biomarkers more specifically associated with other neurodegenerative disorders can also aid in the diagnosis of AD.

\section{Biomarker associations with cognitive function and decline}

Some diagnostic biomarkers may reflect severity of cognitive impairment and thus be useful in disease staging. To assess this, we correlated CSF biomarker levels with MMSE scores at the time of CSF collection as a general measure of cognitive impairment. Among CSF biomarkers for AD identified by at least one approach, six (C3, CgA, IL-1 $\alpha, \mathrm{I}-309, \mathrm{NrCAM}$ and VEGF) were correlated with MMSE score, and levels of these analytes did not correlate with MMSE scores in the other neurodegenerative disorders. A multivariate linear regression analysis adjusting for age, gender and education showed C3, IL-1 $\alpha$ and I-309 levels were independently associated with MMSE scores in autopsy-confirmed cases of AD.

To further test the value of these CSF biomarkers in predicting cognitive decline, we determined the correlation between levels of these six biomarkers and rates of subsequent MMSE decline in MCI subjects following CSF collection. The 38 living MCI patients were similar to AD patients in age ( 71.39 vs. 70.79 yo, $P=0.674$ ), education (15.66 vs. 14.64 yo, $P=0.143$ ) and gender (42.1 vs 53.0\% women), but MCI patients had higher MMSE scores (mean 26.16, $\mathrm{SD}=2.00$ ) as compared to $\mathrm{AD}$ (mean $17.55, \mathrm{SD}=8.57, P<0.01$ ). The MCI patients had a median follow-up of 52 months (range 30-129 months) and a median rate of MMSE decline of 1.2 points per year (mean 2.0, $\mathrm{SD}=2.0$ ). Among analytes associated with cognitive performance at the time of CSF collection in AD, IL-1 $\alpha$ levels correlated with the rates of MMSE decline $(P=0.003)$, although with modest effect on decline rates $(R=0.498$ for model). A search across 4 traditional and $106 \mathrm{MAP}$ analytes additionally identified thymusexpressed chemokine (TECK) as significantly associated with rates of cognitive decline in MCI $(P<0.001$ adjusting for age, gender and education) and had a stronger effect on the rate of decline $(R=0.745$ for model, Fig. 4$)$.

\section{Discussion}

The search for accurate CSF and plasma biomarkers in neurodegenerative diseases has intensified with the increasing need for informative biomarkers in clinical trials of disease- 
modifying therapies for $\mathrm{AD}$, and has been facilitated by high-throughput multiplex platforms $[21,24]$. Using clinically and pathologically well-characterized cases of AD and FTLD, we identified novel biomarkers useful in improving the distinction between AD and cognitively normal subjects, such as NrCAM and PDGF and biomarkers associated with other disorders that improved the classification between $\mathrm{AD}$ and non- $\mathrm{AD}$ dementia, including AgRP, eotaxin-3 and HGF. C3, IL- $1 \alpha$ and I-309 were helpful in the staging of AD, and IL- $1 \alpha$ and TECK levels correlated with rates of subsequent cognitive decline in MCI. We discuss these findings below.

In none of our analytical models did MAP biomarkers alone out-perform traditional AD biomarkers in identifying $\mathrm{AD}$ from non-AD cases, but they complemented traditional biomarkers in two ways. First, while decreased A $\beta 42$ and increased total/phosphorylated-tau levels are strongly linked to $\mathrm{AD}$, altered levels of some MAP biomarkers improved the classification of cognitively normal subjects with decreased levels of CSF A $\beta$, but no dementia. Alterations in MAP biomarkers (NrCAM, PDGF) were seen in multiple neurodegenerative disorders, and likely represent neuronal loss rather than AD-specific processes (Fig. 1). $\mathrm{NrCAM}$ is a member of the L1 family of cell adhesion molecules, and may be involved in ion channel clustering at the axon initial segment and nodes of Ranvier [4,5]. A decrease in NrCAM levels in $\mathrm{AD}$ and other neurodegenerative disorders likely follows axonal degeneration, although accumulation of ankryn (which interacts with NrCAM intracellularly) in the insoluble AD proteome relative to normal and FTLD-TDP brains raises additional possibilities [10]. PDGF was previously identified as a plasma AD biomarker by Ray et al. [21]. PDGF-receptor activation can promote $A \beta$ precursor protein processing in vitro [9], and inhibition of PDGFreceptor activation with imatinib mesylate can decrease $A \beta 40$ and $A \beta 42$ secretion [17]. In our cohort, PDGF was also found to be elevated in multiple disorders, and its constitutive expression by neurons [8] suggests elevated PDGF levels to also reflect more general neuronal loss. $\mathrm{C} 3$ and Fabp were identified as AD biomarkers by two algorithms, and CSF Fabp was elevated in AD and DLB cases in one other study [25]. Despite alterations in multiple forms of dementia, however, changes in these biomarkers associated with neuronal loss improved the distinction between $\mathrm{AD}$ and cognitively normal with age-associated amyloidosis by traditional AD biomarkers alone, and they can further serve as secondary endpoints in therapies aimed at $\mathrm{A} \beta 42$ or tau clearance.

In addition to traditional AD CSF biomarkers (i.e. tau and A $\beta 42$ ), altered levels of resistin and thrombospondin-1 specifically associated with AD despite little classification value beyond analytes in Fig. 2a. Central resistin modulates leptin action and oral intake [19], and resistin as a marker of macrophage may mean preferential microglial activation. Thrombospondin-1 is a key molecule in astrocyte-induced neurogenesis [14], and can promote recovery after brain ischemia. Elevated thrombospondin-1 levels in a subgroup of AD patients may identify a unique subgroup with vascular and degenerative etiologies for their dementia. Further stratification of AD patients by their CSF resistin and/or thrombospondin-1 levels in a larger cohort should clarify their role in AD lesions.

Novel MAP biomarkers also represent candidate biomarkers of disease staging and prediction of progression. Cross-sectionally, six diagnostic AD biomarkers correlated with cognitive deficits at the time of CSF collection. Because these analytes likely mirror severity of neurodegeneration, correlations between their levels and clinical status should be expected.

Furthermore, IL-1 $\alpha$ levels were modestly associated with rates of decline in MCI after the CSF was collected. IL-1 $\alpha$ immunoreactive microglia in AD neuritic plaques have been implicated in plaque evolution [11], and the difference in IL- $1 \alpha$ levels between fast and slow MCI decliners may signal cognitive deficits insensitive to MMSE alone. We also identified TECK to predict the rate of cognitive decline among MCI patients, even though TECK itself was not a robust diagnostic biomarker for $\mathrm{AD}$. This can be due to the potential pathologic heterogeneity of MCI, or represent a biomarker change that is transient in nature and specifically linked to the MCI 
or pre-AD stage. TECK (CCL25) is best understood as a strong chemoattractant for thymocytes and intestinal T cells [15]. TECK is a ligand to CCR9 which is predominantly expressed in epithelial tissues, but also a ligand to CCX-CKR found in the human brain [27,31]. The role of TECK in AD has never been investigated, and its role as a robust predictor of cognitive decline in MCI should prompt further examination of its role in AD.

Some analytes were identified by only one analytical strategy as a potential AD biomarker due to the non-uniqueness of multiple analytical strategies, begging the question of whether such analytes are "true" biomarkers. Notably, the number of ApoE4 alleles was only identified by one analytical strategy (logistic regression, data not shown) to be a significant predictor of $\mathrm{AD}$ versus cognitively normal, despite its known association with $\mathrm{AD}[23]$. The ordinal nature of allele dosage (such as number of ApoE4 alleles) may be more suited for models using linear scaling and less preferred by random forest and PAM. Consequently, we elected to seek analytes of wider ranging levels as novel biomarkers for more uniformity among the algorithms. Among other analytes identified only by one algorithm, IL-1 $\alpha$ appears to be important in disease staging, and HGF was previously found to differentiate between AD and PSP [28]. Several explanations are possible. First, some analytes may correlate strongly with others, and each strategy may select different proxy analytes to represent a group of correlated analytes from the same biological process. Second, different analytical strategies may have various strengths and weaknesses for detecting particular effects. This was the reason we chose three analytical strategies to identify putative AD biomarkers, and analytes identified by multiple strategies may be most reliable. Third, some analytes identified by only one analytical strategy may be associated with chance difference at the population level not directly associated with dementia or $\mathrm{AD}$. These speculations notwithstanding, each putative novel biomarker's value in diagnosis and prognosis needs independent validation in another single-or a multicenter study, and their biological significance should be assessed independently. Indeed, we have studies underway now to do exactly this.

In summary, we identified novel biomarkers associated with pathologically confirmed AD. Some analytes were specifically associated with AD including A $\beta 42$, resistin, and thrombospondin-1, while others were associated with multiple neurodegenerative disorders. Some diagnostic biomarkers mirrored the severity of cognitive impairment at time of CSF collection, while TECK and IL- $1 \alpha$ reflected the rate of cognitive decline among clinically diagnosed MCI subjects. Accordingly, we propose the inclusion of diagnostic and prognostic biomarkers in a composite AD biomarker panel. Given the variability of each candidate biomarker across individuals, their collective classifying power should be determined in a large multicenter cohort, such as the Alzheimer Disease Neuroimaging Initiative. The biological relevance of each individual and set of biomarkers should be investigated for potential targets of therapeutic developments.

\section{Supplementary Material}

Refer to Web version on PubMed Central for supplementary material.

\section{Acknowledgments}

This work has been supported by the Penn-Pfizer Alliance as well as AG-10124 and AG-17586. WTH is supported by the American Academy of Neurology Clinical Translational Research Fellowship. ACP is supported by a Burroughs Wellcome Fund Career Award for Medical Scientists and NIH K08 AG033101. EP, MK, YC, and HDS are employees of Pfizer Global Research and Development. 


\section{References}

1. Castano EM, Roher AE, Esh CL, Kokjohn TA, Beach T. Comparative proteomics of cerebrospinal fluid in neuropathologically-confirmed Alzheimer's disease and non-demented elderly subjects. Neurol Res 2006;28:155-163. [PubMed: 16551433]

2. Clark CM, Davatzikos C, Borthakur A, et al. Biomarkers for early detection of Alzheimer pathology. Neurosignals 2008;16:11-18. [PubMed: 18097155]

3. Clark CM, Xie S, Chittams J, et al. Cerebrospinal fluid tau and beta-amyloid: how well do these biomarkers reflect autopsy-confirmed dementia diagnoses? Arch Neurol 2003;60:1696-1702. [PubMed: 14676043]

4. Custer AW, Kazarinova-Noyes K, Sakurai T, et al. The role of the ankyrin-binding protein Nrcam in node of Ranvier formation. J Neurosci 2003;23:10032-10039. [PubMed: 14602817]

5. Davis JQ, Bennett V. Ankyrin binding activity shared by the Neurofascin/L1/Nrcam family of nervous system cell adhesion molecules. J Biol Chem 1994;269:27163-27166. [PubMed: 7961622]

6. Dubois B, Feldman HH, Jacova C, et al. Research criteria for the diagnosis of Alzheimer's disease: revising the Nincds-Adrda criteria. Lancet Neurol 2007;6:734-746. [PubMed: 17616482]

7. Finehout EJ, Franck Z, Choe LH, Relkin N, Lee KH. Cerebrospinal fluid proteomic biomarkers for Alzheimer's disease. Ann Neurol 2007;61:120-129. [PubMed: 17167789]

8. Fruttiger M, Calver AR, Richardson WD. Platelet-derived growth factor is constitutively secreted from neuronal cell bodies but not from axons. Curr Biol 2000;10:1283-1286. [PubMed: 11069109]

9. Gianni D, Zambrano N, Bimonte M, et al. Platelet-derived growth factor induces the beta-gammasecretase-mediated cleavage of Alzheimer's amyloid precursor protein through a Src-Rac-dependent pathway. J Biol Chem 2003;278:9290-9297. [PubMed: 12645527]

10. Gozal YM, Duong DM, Gearing M, et al. Proteomics analysis reveals novel components in the detergent-insoluble subproteome in Alzheimer's disease. J Proteome Res 2009;8:5069-5079. [PubMed: 19746990]

11. Griffin WS, Sheng JG, Roberts GW, Mrak RE. Interleukin-1 expression in different plaque types in Alzheimer's disease: significance in plaque evolution. J Neuropathol Exp Neurol 1995;54:276-281. [PubMed: 7876895]

12. Grossman M, Farmer J, Leight S, et al. Cerebrospinal fluid profile in frontotemporal dementia and Alzheimer's disease. Ann Neurol 2005;57:721-729. [PubMed: 15852395]

13. Lippa CF, Duda JE, Grossman M, et al. Dlb and Pdd boundary issues: diagnosis, treatment, molecular pathology, and biomarkers. Neurology 2007;68:812-819. [PubMed: 17353469]

14. Lu Z, Kipnis J. Thrombospondin 1-a key astrocyte-derived neurogenic factor. Faseb J.

15. Moser B, Wolf M, Walz A, Loetscher P. Chemokines: multiple levels of leukocyte migration control. Trends Immunol 2004;25:75-84. [PubMed: 15102366]

16. Neary D, Snowden JS, Gustafson L, et al. Frontotemporal lobar degeneration: a consensus on clinical diagnostic criteria. Neurology 1998;51:1546-1554. [PubMed: 9855500]

17. Netzer WJ, Dou F, Cai D, et al. Gleevec inhibits beta-amyloid production but not notch cleavage. Proc Natl Acad Sci USA 2003;100:12444-12449. [PubMed: 14523244]

18. Neumann M, Kwong LK, Lee EB, et al. Phosphorylation of S409/410 of Tdp-43 is a consistent feature in all sporadic and familial forms of Tdp-43 proteinopathies. Acta Neuropathol 2009;117:137-149. [PubMed: 19125255]

19. Park S, Hong SM, Sung SR, Jung HK. Long-term effects of central leptin and resistin on body weight, insulin resistance, and beta-cell function and mass by the modulation of hypothalamic leptin and insulin signaling. Endocrinology 2008;149:445-454. [PubMed: 17991727]

20. Peskind ER, Li G, Shofer J, et al. Age and apolipoprotein E*4 allele effects on cerebrospinal fluid beta-amyloid 42 in adults with normal cognition. Arch Neurol 2006;63:936-939. [PubMed: 16831961]

21. Ray S, Britschgi M, Herbert C, et al. Classification and prediction of clinical Alzheimer's diagnosis based on plasma signaling proteins. Nat Med 2007;13:1359-1362. [PubMed: 17934472]

22. Ross MA, Miller RG, Berchert L, et al. Toward earlier diagnosis of amyotrophic lateral sclerosis: revised criteria: Rhcntf Als Study Group. Neurology 1998;50:768, 772. [PubMed: 9521272] 
23. Shaw LM, Korecka M, Clark CM, Lee VM, Trojanowski JQ. Biomarkers of neurodegeneration for diagnosis and monitoring therapeutics. Nat Rev Drug Discov 2007;6:295-303. [PubMed: 17347655]

24. Shaw LM, Vanderstichele H, Knapik-Czajka M, et al. Cerebrospinal fluid biomarker signature in Alzheimer's disease neuroimaging initiative subjects. Ann Neurol 2009;65:403-413. [PubMed: 19296504]

25. Steinacker P, Mollenhauer B, Bibl M, et al. Heart fatty acid binding protein as a potential diagnostic marker for neurodegenerative diseases. Neurosci Lett 2004;370:36-39. [PubMed: 15489013]

26. Tapiola T, Alafuzoff I, Herukka SK, et al. Cerebrospinal fluid \{beta\}-amyloid 42 and tau proteins as biomarkers of alzheimer-type pathologic changes in the brain. Arch Neurol 2009;66:382-389. [PubMed: 19273758]

27. Townson JR, Nibbs RJ. Characterization of mouse Ccx-Ckr, a receptor for the lymphocyte-attracting chemokines Teck/Mccl25, Slc/Mccl21 and Mip-3beta/Mccl19: comparison to human CCX-Ckr. Eur J Immunol 2002;32:1230-1241. [PubMed: 11981810]

28. Tsuboi Y, Kakimoto K, Nakajima M, et al. Increased hepatocyte growth factor level in cerebrospinal fluid in Alzheimer's disease. Acta Neurol Scand 2003;107:81-86. [PubMed: 12580855]

29. Tusher VG, Tibshirani R, Chu G. Significance analysis of microarrays applied o the ionizing radiation response. Proc Natl Acad Sci USA 2001;98:5116-5121. [PubMed: 11309499]

30. Winblad B, Palmer K, Kivipelto M, et al. Mild cognitive impairment-beyond controversies, towards a consensus: report of the international working group on mild cognitive impairment. J Intern Med 2004;256:240-246. [PubMed: 15324367]

31. Youn BS, Yu KY, Oh J, Lee J, Lee TH, Broxmeyer HE. Role of the Cc chemokine receptor 9/Teck interaction in apoptosis. Apoptosis 2002;7:271-276. [PubMed: 11997671] 


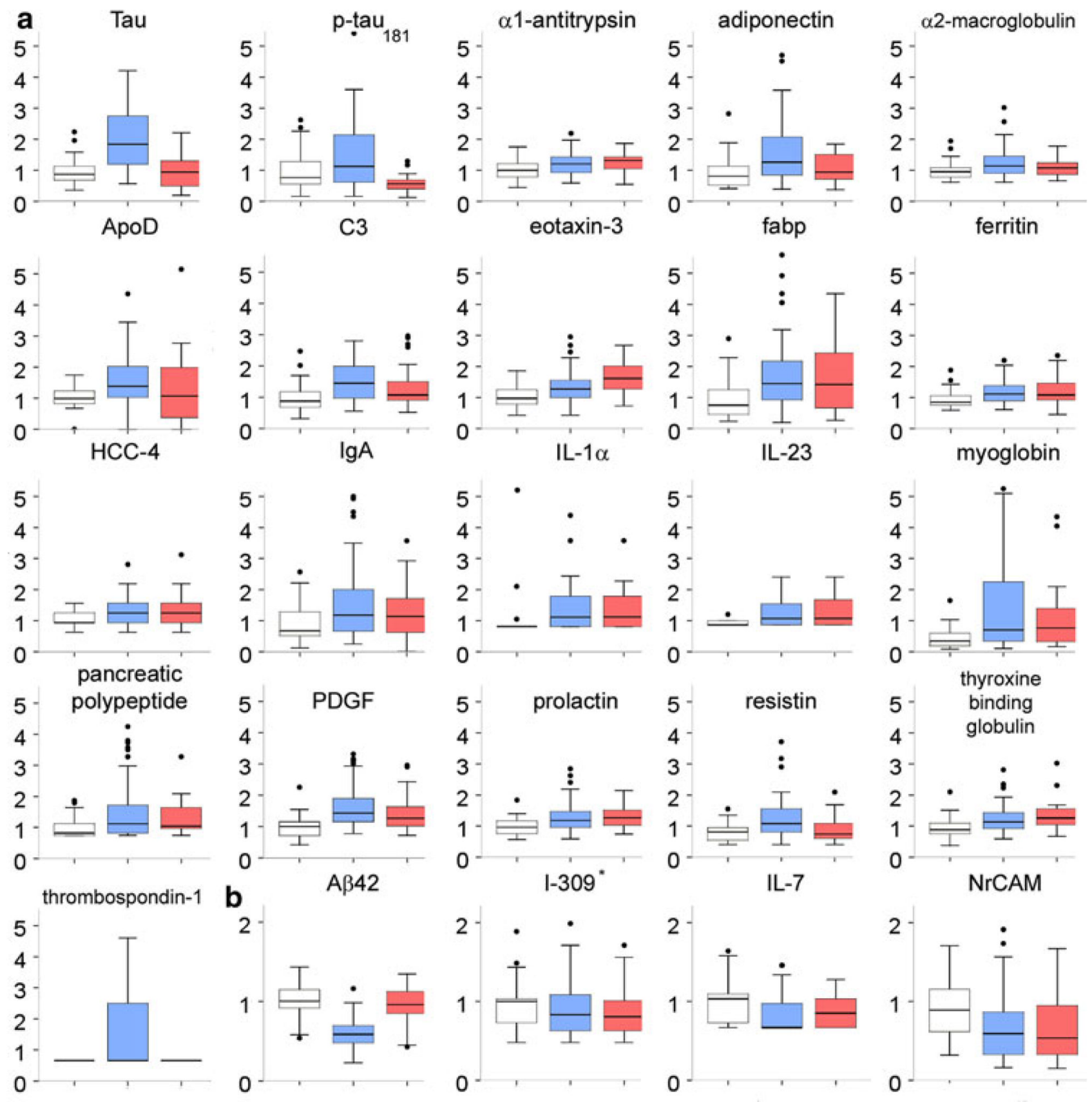

Fig. 1.

Boxplots showing median values, quartiles, and outliers (circles) of traditional (i.e. tau and $\mathrm{A} \beta 42$ ) and other candidate CSF biomarkers that differed in levels between subjects with normal cognition and AD. Values shown are normalized to mean values of cognitively normal subjects. a Analytes elevated in AD as compared to cognitively normal subjects. b Analytes decreased in AD as compared to cognitively normal subjects. Levels in patients with autopsy-confirmed non-AD neurodegeneration were also shown for comparison. White box cognitively normal subjects, blue box autopsy-confirmed cases of AD, red box autopsy-confirmed cases of nonAD neurodegenerative disorders. *I-309 was found to differ between AD and cognitively normal subjects by random forest and PAM, but not Mann-Whitney $U$ test 

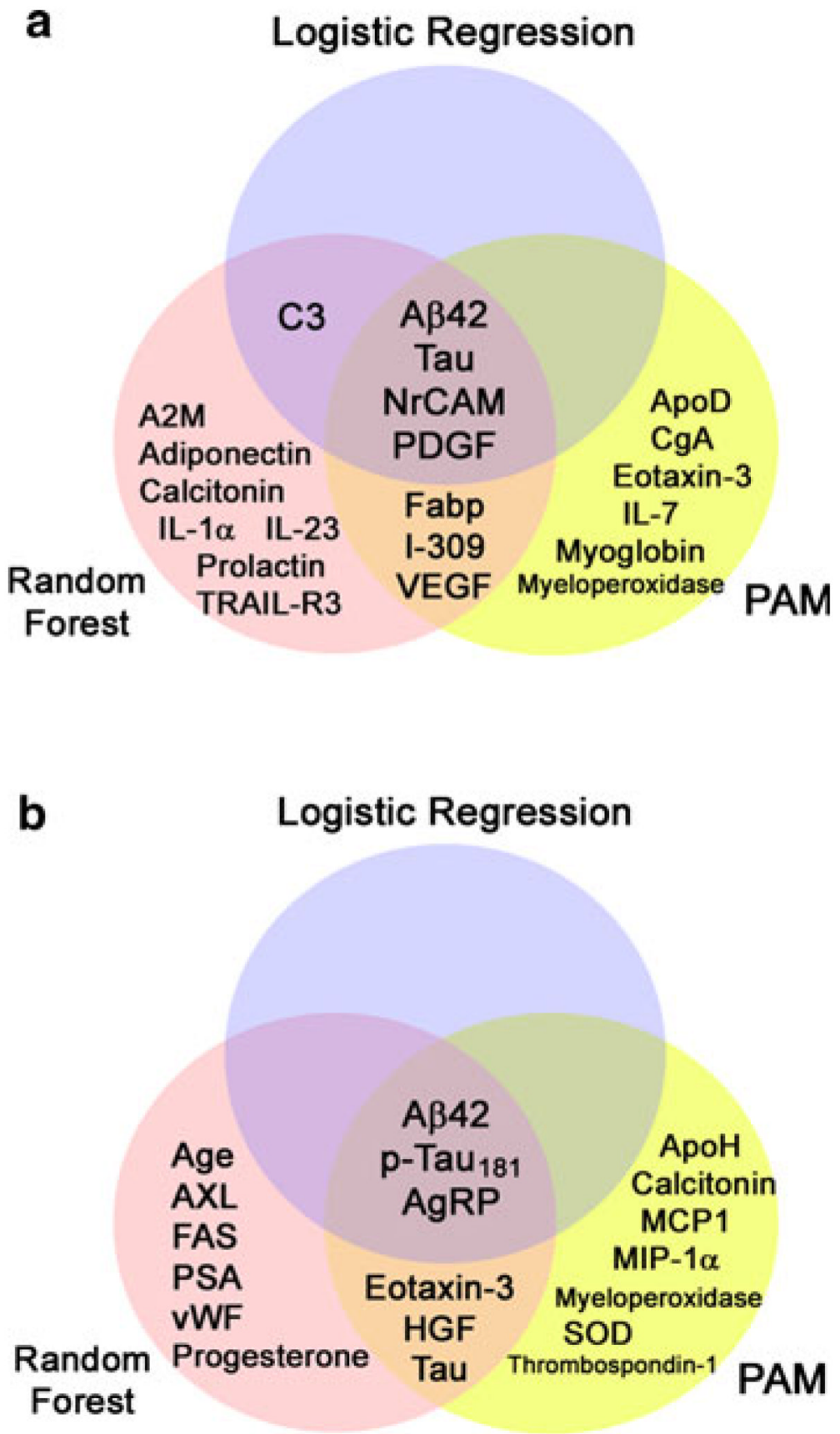

Fig. 2.

AD biomarkers identified by each of the three analytical strategies (logistic regression, random forest, and PAM). a Biomarkers useful in distinguishing between subjects with AD and normal cognition. b Biomarkers useful in distinguishing between subjects with AD and other non-AD neurodegenerative disorders. Analytes in overlapping regions were identified by multiple strategies as important biomarkers 

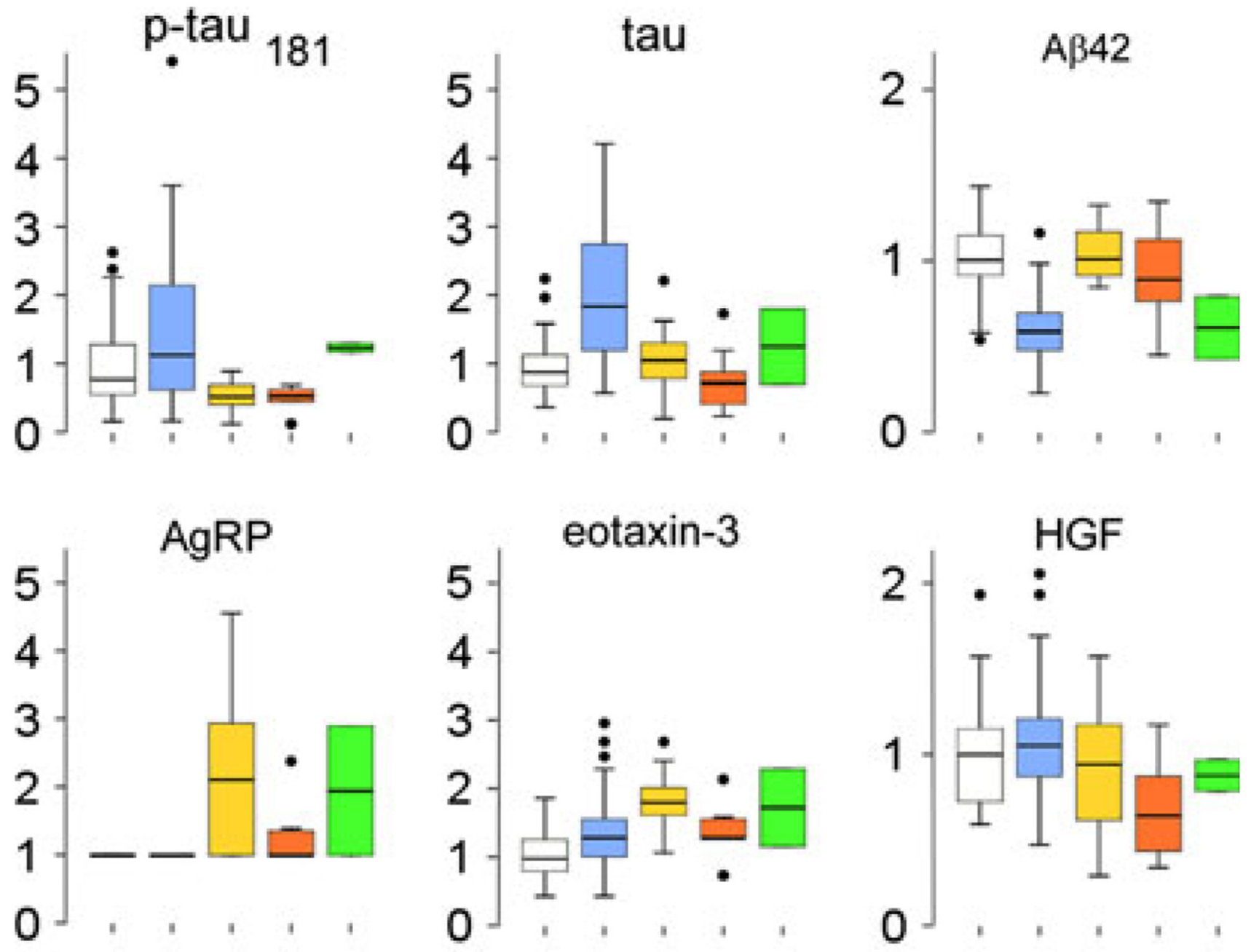

Fig. 3.

Boxplots showing median values, quartiles, and outliers (circles) of traditional and candidate biomarkers that differed in levels between $\mathrm{AD}$ and other non-AD neurodegenerative disorders. Values shown are normalized to mean values of cognitively normal subjects. White box cognitively normal subjects, blue box AD, yellow box FTLD-TDP, orange box FTLD-Tau, green box dementia with Lewy bodies 

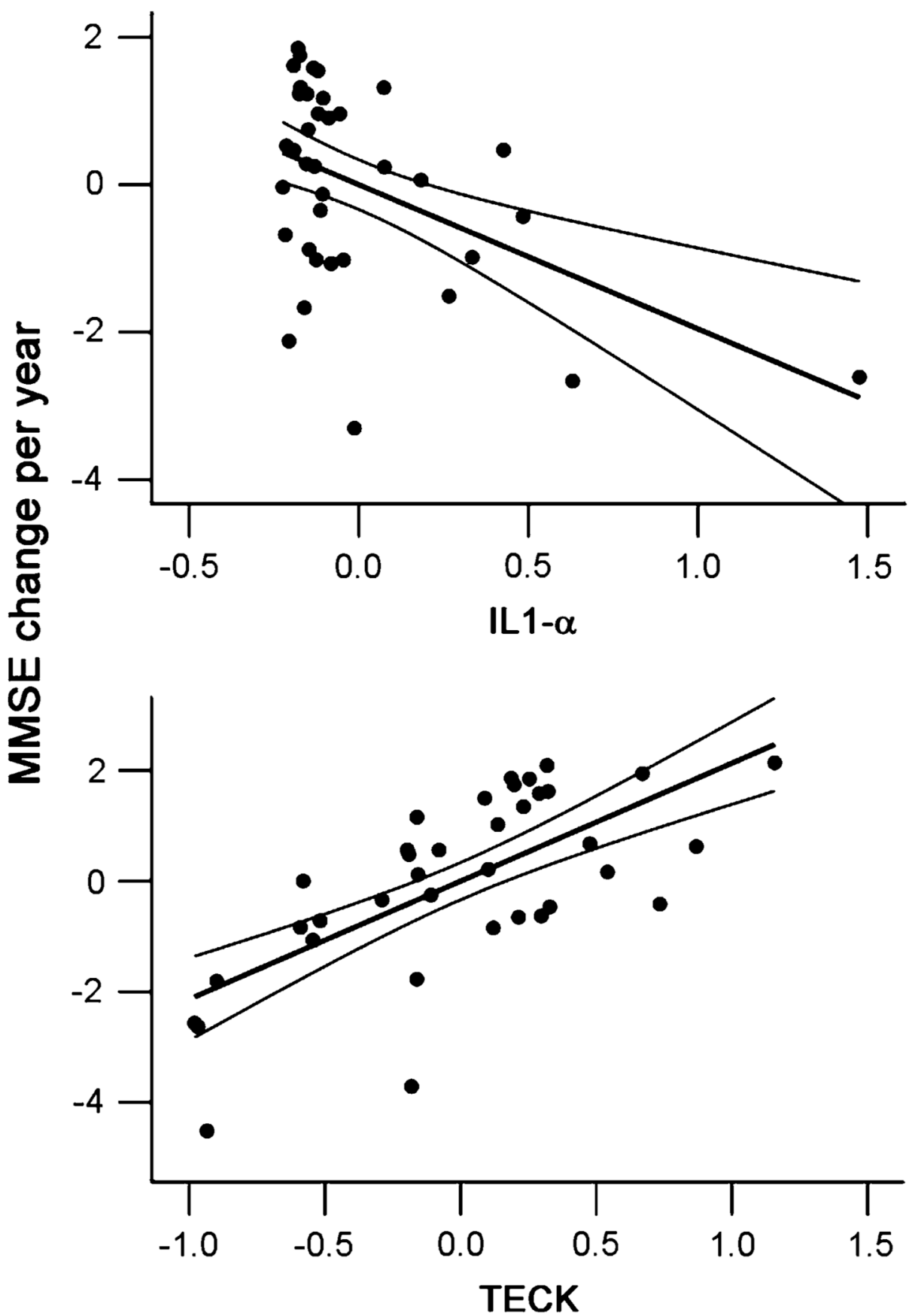

Fig. 4.

Partial residual plots of MAP analytes versus rates of subsequent cognitive decline in MCI. Linear fit and 95\% confidence interval for fit are shown for each graph. The overall model includes age, gender, education, IL-1 $\alpha$ level and TECK level 


\section{Table 1}

Factors predictive of AD compared with cognitively normal subjects according to logistic regression

\begin{tabular}{lrr}
\hline AD versus cognitively normal & $\boldsymbol{B}$ & $\boldsymbol{P}$ \\
\hline MAP model & -0.073 & 0.141 \\
Age & 1.845 & 0.092 \\
Male gender & 0.932 & 0.017 \\
C3 & 0.809 & 0.002 \\
Fabp & 17.24 & 0.031 \\
IL-23 & -0.051 & $<0.001$ \\
NrCAM & 0.004 & 0.064 \\
PDGF & & \\
Traditional AD model & -0.024 & 0.558 \\
Age & 0.001 & 0.999 \\
Male gender & -0.035 & $<0.001$ \\
A $\beta 42$ & 0.019 & 0.051 \\
Tau & & \\
Combined model & -0.217 & 0.088 \\
Age & 2.038 & 0.309 \\
Male gender & 2.376 & 0.025 \\
C3 & -0.063 & 0.041 \\
NrCAM & 0.013 & 0.061 \\
PDGF & 0.08 & 0.042 \\
Tau & -0.039 & 0.01 \\
A $\beta 42$ & &
\end{tabular}

Traditional AD model incorporated $\mathrm{A} \beta 42$ and tau levels

Coefficient $(B)$ and $P$ value for each factor as part of the overall model are shown

Age and gender were entered into first block of LR, while analytes identified to be different between AD and cognitively normal subjects were then entered in a forward step-wise fashion, with $P<0.05$ for entry and $P>0.10$ for removal 
Table 2

Analytes differentiating AD from cognitively normal according to random forest analysis

\begin{tabular}{|c|c|}
\hline $\begin{array}{l}\text { AD versus } \\
\text { cognitively normal }\end{array}$ & $Z$ score \\
\hline \multicolumn{2}{|l|}{ MAP model } \\
\hline PDGF & 27.585 \\
\hline IL- $1 \alpha$ & 26.656 \\
\hline IL-23 & 20.686 \\
\hline $\mathrm{C} 3$ & 18.343 \\
\hline Fabp & 17.888 \\
\hline $\mathrm{NrCAM}$ & 15.937 \\
\hline VEGF & 13.894 \\
\hline TRAIL-R3 & 12.064 \\
\hline IL-17 & 11.209 \\
\hline Eotaxin-3 & 11.037 \\
\hline IL-7 & 10.48 \\
\hline $\mathrm{A} 2 \mathrm{M}$ & 9.603 \\
\hline Prolactin & 9.549 \\
\hline Ferritin & 8.463 \\
\hline ThBG & 8.371 \\
\hline I-309 & 7.743 \\
\hline HCC-4 & 3.502 \\
\hline \multicolumn{2}{|l|}{ Traditional AD model } \\
\hline $\mathrm{A} \beta 42$ & 68.401 \\
\hline Tau & 21.826 \\
\hline Male gender & 10.052 \\
\hline \multicolumn{2}{|l|}{ Combined model } \\
\hline $\mathrm{A} \beta 42$ & 47.935 \\
\hline Tau & 26.232 \\
\hline PDGF & 22.506 \\
\hline IL-1 $\alpha$ & 19.806 \\
\hline $\mathrm{NrCAM}$ & 17.474 \\
\hline $\mathrm{C} 3$ & 16.702 \\
\hline IL-23 & 14.472 \\
\hline VEGF & 13.362 \\
\hline Fabp & 11.583 \\
\hline Prolactin & 11.533 \\
\hline TRAIL-R3 & 9.779 \\
\hline $\mathrm{A} 2 \mathrm{M}$ & 9.425 \\
\hline $\mathrm{I}-309$ & 7.981 \\
\hline Calcitonin & 5.824 \\
\hline Adiponectin & 4.64 \\
\hline
\end{tabular}

Acta Neuropathol. Author manuscript; available in PMC 2010 June 4. 
Table 3

Factors differentiating AD from cognitively normal according to PAM

\begin{tabular}{|c|c|c|}
\hline MAP model & $\begin{array}{l}\text { Traditional } \\
\text { AD model }\end{array}$ & Combined model \\
\hline \multicolumn{3}{|c|}{$\mathrm{AD}$ versus cognitively normal } \\
\hline PDGF & $\mathrm{A} \beta 42$ & $\mathrm{~A} \beta 42$ \\
\hline VEGF & Tau & Tau \\
\hline NrCAM & $\mathrm{p}$-tau 181 & PDGF \\
\hline $\mathrm{CgA}$ & Age & VEGF \\
\hline ApoD & & NrCAM \\
\hline Fabp & & $\mathrm{CgA}$ \\
\hline $\mathrm{I}-309$ & & ApoD \\
\hline Eotaxin-3 & & Fabp \\
\hline IL-7 & & Eotaxin-3 \\
\hline Myoglobin & & I-309 \\
\hline Myeloperoxidase & & IL-7 \\
\hline GRO- $\alpha$ & & Myoglobin \\
\hline EN-RAGE & & Myeloperoxidase \\
\hline \multicolumn{3}{|l|}{ TGF $\alpha$} \\
\hline \multicolumn{3}{|l|}{ Thrombospondin-1 } \\
\hline \multicolumn{3}{|l|}{ Age } \\
\hline \multicolumn{3}{|l|}{ Stem cell factor } \\
\hline \multicolumn{3}{|l|}{ Tissue factor } \\
\hline \multicolumn{3}{|l|}{ Pancreatic polypeptide } \\
\hline \multicolumn{3}{|l|}{ MDC } \\
\hline \multicolumn{3}{|l|}{ TECK } \\
\hline \multicolumn{3}{|l|}{ SOD } \\
\hline \multicolumn{3}{|l|}{ Ferritin } \\
\hline \multicolumn{3}{|l|}{ EGF-R } \\
\hline \multicolumn{3}{|l|}{ IL-11 } \\
\hline \multicolumn{3}{|l|}{ FAS } \\
\hline \multicolumn{3}{|l|}{ IL-1ra } \\
\hline \multicolumn{3}{|l|}{ Prolactin } \\
\hline \multicolumn{3}{|l|}{ AXL } \\
\hline \multicolumn{3}{|l|}{ IL-17 } \\
\hline \multicolumn{3}{|l|}{ TRAIL-R3 } \\
\hline \multicolumn{3}{|l|}{ FAS-ligand } \\
\hline IL-16 & & \\
\hline
\end{tabular}

Threshold was set for each model through internal cross-validation 


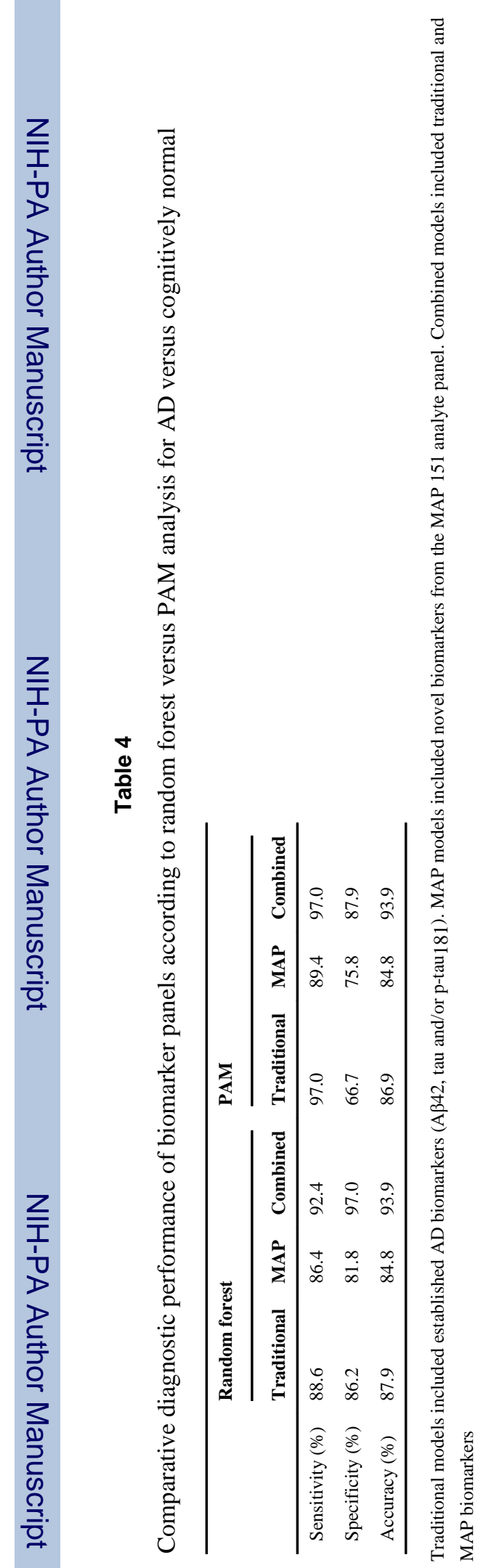

Acta Neuropathol. Author manuscript; available in PMC 2010 June 4. 